Volume 69, Number 2, Pages 1070-1082 (2020)

DOI: $10.31801 /$ cfsuasmas. 620674

ISSN 1303-5991 E-ISSN 2618-6470

Received by the editors: September 16, 2019; Accepted: May 08, 2020

\title{
DIGITAL HAUSDORFF DISTANCE ON A CONNECTED DIGITAL IMAGE
}

\author{
Tane VERGILI \\ Karadeniz Technical University, Faculty of Science Department of Mathematics Trabzon, \\ TURKEY
}

\begin{abstract}
A digital image $X$ can be considered as a subset of $\mathbb{Z}^{n}$ together with an adjacency relation where $\mathbb{Z}$ is the set of the integers and $n$ is a natural number. The aim of this study is to measure the closeness of two subsets of a connected digital image. To do this, we adapt the Hausdorff distance in the topological setting to its digital version. In this paper, we define a metric on a connected digital image by using the length of the shortest digital simple path. Then we use this metric to define the $r$-thickening of the subsets of a connected digital image and define the digital Hausdorff distance between them.
\end{abstract}

\section{INTRODUCTION}

Digital images can be considered to be subsets of $\mathbb{Z}^{n}$. We study digital images to analyze not only their features but also their correlations with the others. Investigating the features of the digital images by their topological properties would be fine but the problem here is that the digital images are not topological spaces, they are just sets. This problem can be achieved by imposing an adjacency relation on a digital image to adapt the topological concepts such as neighborhood, continuity, connectivity, homotopy, and contractibility to their digital versions.

The distance between two points in a connected digital image is obtained via the shortest simple path metric which is denoted by $d^{\kappa}$. Then the distance, called a digital Hausdorff distance, between two subsets of a connected digital image can be calculated via $d^{\kappa}$ as follows: One starts with fattening each subset by taking the union of the neighborhoods with radius $r$ of all its points. We call this new set the $r$-thickening of a subset. Then the distance between subsets is the minimum

2020 Mathematics Subject Classification. Primary 54H99, 68R01; Secondary 68U10.

Keywords and phrases. Digital topology, Hausdorff distance, digital image.

tane.vergili@ktu.edu.tr

(D) 0000-0003-1821-6697.

(C)2020 Ankara University Communications Faculty of Sciences University of Ankara-Series A1 Mathematics and Statistics 
$r$ such that the $r$-thickening of both include another. Like the Hausdorff distance in topological setting [20], the digital Hausdorff distance will measure how close the two subsets are to each other. Note that the distance may differ according to the adjacency relation defined on a digital image. We also investigate that assigning each point to its neighborhood with radius $r$ leads to a strongly (hence a weakly) continuous multi-valued function from a connected digital image to itself (Theorem 6). We observe that the image of the $r$-thickening of a given subset under a digital continuous map is contained in the $r$-thickening of the image of that subset (Theorem 14). We also show that the digital Hausdorff distance between the image of two subsets of a connected digital image under a continuous map is less than or equal to the Hausdorff distance between these two subsets (Theorem 24).

\section{BACKGROUND}

To study the features of the digital images, we start with an adjacency relation defined on the points of $\mathbb{Z}^{n}$ to adapt the fundamental concepts of topology. For an integer $\ell$ with $1 \leq \ell \leq n$, we say two distinct points $x=\left(x_{1}, x_{2}, \ldots, x_{2}\right)$ and $y=\left(y_{1}, y_{2}, \ldots, y_{n}\right)$ in $\mathbb{Z}^{n}$ are $c_{\ell}$-adjacent $[11]$ if

i): $\left|x_{i}-y_{i}\right|=1$ for at most $\ell$ indices $i$ and

ii): for all indices $j$ such that $\left|x_{j}-y_{j}\right| \neq 1$, we have $x_{j}=y_{j}$.

It turns out that $c_{1}$-adjacency in $\mathbb{Z}$ is 2-adjacency, $c_{1}$ and $c_{2}$ adjacencies in $\mathbb{Z}^{2}$ are 4 -adjacency and 8-adjacency, and $c_{1}, c_{2}$ and $c_{3}$-adjacencies in $\mathbb{Z}^{3}$ are 6-adjacency, 18 -adjacency and 26-adjacency respectively.

A digital image $X$ is a subset of $\mathbb{Z}^{n}$ for some natural number $n$ together with an adjacency relation $\kappa$ inherited from $\mathbb{Z}^{n}$ and represented by $(X, \kappa)$.

The continuous functions between the digital images are defined in terms of adjacency relations. Let $(X, \kappa)$ and $(Y, \lambda)$ be digital images and $f: X \rightarrow Y$ be a function. Then we say that $f$ is $(\kappa, \lambda)$-continuous iff $f(x)=f\left(x^{\prime}\right)$ or $f(x)$ and $f\left(x^{\prime}\right)$ is $\lambda$-adjacent whenever $x$ and $x^{\prime}$ are $\kappa$-adjacent for $x, x^{\prime} \in X[2]$.

Consider the digital interval

$$
[a, b]_{\mathbb{Z}}=\{c \in \mathbb{Z}: a \leq c \leq b\}
$$

for integers $a, b$ with $a<b$ where 2-adjacency relation is assumed [1]. Then a $\kappa$ path from $x$ to $y$ in a digital image $(X, \kappa)$ is a sequence $\left(x=x_{0}, x_{1}, x_{2}, \ldots, x_{m}=y\right)$ in $X$ such that $x_{i}$ is $\kappa$-adjacent to $x_{i+1}$ for $m \geq 1$ and $0 \leq i \leq m-1$ [26]. In that case, $m$ denotes the length of this path. A $\kappa$-path can be also considered as a $(2, \kappa)$-continuous map $\alpha:[0, m]_{\mathbb{Z}} \rightarrow X$ such that $\alpha(0)=x$ and $\alpha(m)=y[16]$. Note that such a $\kappa$-path $\alpha$ from $x$ to $y$ can be reversed and the resulting map $\bar{\alpha}$, which is explicitly defined by $\bar{\alpha}(t):=\alpha(m-t)$ for $t \in[0, m]_{\mathbb{Z}}$, is a $\kappa$-path from $y$ to $x$. We say that a $\kappa$-path $\left(x=x_{0}, x_{1}, x_{2}, \ldots, x_{m-1}=y\right)$ from $x$ to $y$ is simple, 
provided $x_{i}$ and $x_{j}$ are $\kappa$-adjacent if and only if either $j=i+1, i \in[0, m-2]_{\mathbb{Z}}$ or $i=j+1, j \in[0, m-2]_{\mathbb{Z}}[11,12]$. A digital image $(X, \kappa)$ is said to be $\kappa$-connected, provided there exists a $\kappa$-path between any pair of elements in $X$ [15].

The digital analogue of homotopy is given as follows [2,16]: Let $(X, \kappa)$ and $(Y, \lambda)$ be two digital images and $f, g: X \rightarrow Y$ be $(\kappa, \lambda)$-continuous maps. Then we say $f$ and $g$ are digitally $(\kappa, \lambda)$-homotopic if there is a positive integer $m$ and a function

$$
H: X \times[0, m]_{\mathbb{Z}} \rightarrow Y
$$

such that

i): $H(x, 0)=f(x)$ and $H(x, m)=g(x)$;

ii): for each $t \in[0, m]_{\mathbb{Z}}$, the induced map $H_{t}: X \rightarrow Y$ defined by $H_{t}(x)=$ $H(x, t)$ is $(\kappa, \lambda)$-continuous; and

iii): for each $x \in X$, the induced map $H_{x}:[0, m]_{\mathbb{Z}} \rightarrow Y$ defined by $H_{x}(t)=$ $H(x, t)$ for $t \in[0, m]_{\mathbb{Z}}$ is $(2, \lambda)$-continuous.

Such a function $H$ is called a digital $(\kappa, \lambda)$-homotopy between $f$ and $g$. We denote $f \simeq_{\kappa, \lambda} g$ if there exists a digital $(\kappa, \lambda)$-homotopy between them.

\section{REsults}

3.1. The shortest digital path. Suppose $X$ is a $\kappa$-connected digital image, $x \in X$ and $A$ is a nonempty subset of $X$. Boxer defines $l_{X}^{\kappa}(x, A)$ to be the length of the shortest $\kappa$-path from $x$ to any other point $A$ in [3]. For our purpose, we would want a digital path to be simple. Let $\ell^{\kappa}(x, A)$ denote the length of the shortest simple $\kappa$-path in $X$ from $x$ to any point of $A$. In that case, if $x_{1}$ is another point in $X$ then $\ell^{\kappa}\left(x,\left\{x_{1}\right\}\right)$ turns into its original definition and denotes the length of the shortest simple $\kappa$-path from $x$ to $x_{1}$ given in $[11,12]$. Since any digital path can be reversed, $\ell^{\kappa}\left(x_{0},\left\{x_{1}\right\}\right)=\ell^{\kappa}\left(x_{1},\left\{x_{0}\right\}\right)$ for any pair of elements $x_{0}, x_{1}$ in $X$. Throughout the paper we assume $\ell^{\kappa}(x,\{x\})=0$.

Under the assumption $\ell^{\kappa}(x,\{x\})=0$, consider the function $d^{\kappa}: X \times X \rightarrow \mathbb{R}$ defined by

$$
d^{\kappa}\left(x_{0}, x_{1}\right):=\ell^{\kappa}\left(x_{0},\left\{x_{1}\right\}\right)
$$

for $x_{0}, x_{1}$ in $X$. It's trivial that $d^{\kappa}\left(x_{0}, x_{1}\right)=0$ iff $x_{0}=x_{1}$ and we have $d^{\kappa}\left(x_{0}, x_{1}\right)=$ $d^{\kappa}\left(x_{1}, x_{0}\right)$ since every digital simple $\kappa$-path from $x_{0}$ to $x_{1}$ can be reversed as we mentioned before.

$d^{\kappa}$ also satisfies the triangle inequality

$$
d^{\kappa}\left(x_{0}, x_{1}\right) \leq d^{\kappa}\left(x_{0}, x_{2}\right)+d^{\kappa}\left(x_{2}, x_{1}\right)
$$

for $x_{0}, x_{1}, x_{2} \in X$. Suppose $\alpha:[0, m]_{\mathbb{Z}} \rightarrow X$ and $\beta:[0, k]_{\mathbb{Z}} \rightarrow X$ are simple $\kappa$-paths with shortest lengths from $x_{0}$ to $x_{2}$ and $x_{2}$ to $x_{1}$ respectively. In that case, we 
have $\alpha(0)=x_{0}, \alpha(m)=x_{2}, \beta(0)=x_{2}$, and $\beta(k)=x_{1}$. Then $\gamma:[0, m+k]_{\mathbb{Z}} \rightarrow X$ defined by

$$
\gamma(t)= \begin{cases}\alpha(t), & 0 \leq t \leq i \\ \beta(t-m), & i \leq t \leq m+k\end{cases}
$$

is a $\kappa$-path from $x_{0}$ to $x_{1}$. Note that $\gamma$ may not be simple. Since there always exists a simple $\kappa$-path from $x_{0}$ to $x_{1}$ with a shortest length, say $i$, then $i$ should be less than or equal to $m+k$.

Hence $d^{\kappa}$ is a metric on a connected digital image $X$. We refer to [13,14] for further reading.

Definition 1. We call the metric $d^{\kappa}$ given in (1) the shortest simple $\kappa$-path metric on a connected digital image.

Let $X$ be a $\kappa$-connected digital image and $x_{0} \in X$. The $\kappa$-neighborhood of $x_{0}$ in $X$ with some radius $r \geq 0$ is given by [11]

$$
\mathcal{B}_{\kappa}\left(x_{0}, r\right)=\left\{x \in X: d^{\kappa}\left(x_{0}, x\right) \leq r\right\} .
$$

Obviously, $B_{\kappa}\left(x_{0}, r\right)$ is a $\kappa$-connected subset of $X$ and $\mathcal{B}_{\kappa}\left(x_{0}, 0\right)=\left\{x_{0}\right\}$.

Lemma 2. Suppose $(X, \kappa)$ is a digital image and $x_{1}, x_{2} \in X$. If $x_{1}$ and $x_{2}$ are $\kappa$-adjacent, then $x_{2} \in \mathcal{B}_{\kappa}\left(x_{1}, 1\right)$ and $x_{1} \in \mathcal{B}_{\kappa}\left(x_{2}, 1\right)$.

Proof. The shortest simple $\kappa$-path from $x_{1}$ and $x_{2}$ in $X$ is a path $\alpha:[0,1]_{\mathbb{Z}} \rightarrow X$ with $\alpha(0)=x$ and $\alpha(1)=y$.

A digital image $(X, \kappa)$ is said to be $\kappa$-contractible if its identity function is digitally $(\kappa, \kappa)$-homotopic to a constant map on $X[1]$.

Theorem 3. Suppose $X$ is a $\kappa$-connected digital image and $x \in X$. Then $\mathcal{B}_{\kappa}(x, r)$ is $\kappa$-contractible for $r=1,2$ but $\mathcal{B}_{\kappa}(x, r)$ may not be $\kappa$-contractible for $r \geq 3$.

Proof. To see the contractibility of $\mathcal{B}_{\kappa}(x, r)$ for $r=1,2$, we will construct a digital homotopy between the identity map on it and a constant map at $x$. We define the digital homotopy

$$
H: \mathcal{B}_{\kappa}(x, r) \times[0,1]_{\mathbb{Z}} \rightarrow \mathcal{B}_{\kappa}(x, r)
$$

by $H(y, 0)=y$ and $H(y, 1)=x$ for every $y \in \mathcal{B}_{\kappa}(x, r)$. Then $H$ is the desired homotopy.

Consider the 8-connected digital image

$$
\mathrm{MSC}_{8}=\{(0,0),(1,1),(2,1),(3,0),(2,-1),(1,-1)\}
$$

in $\mathbb{Z}^{2}$ illustrated in Figure 1. Observe that $\mathcal{B}_{8}((0,0), 3)$ is the entire image $\mathrm{MSC}_{8}$ which is not 8-contractible [11]. 


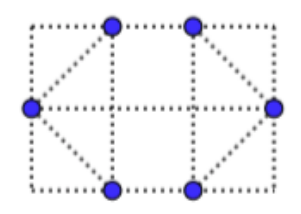

Figure 1. The digital image $\mathrm{MSC}_{8}$ is 8 -connected but not 8contractible [11].

Two subsets $A$ and $B$ of a digital image $(X, \kappa)$ are said to be $\kappa$-adjacent if there exist $a \in A$ and $b \in B$ such that $a=b$ or $a$ and $b$ are $\kappa$-adjacent [3].

Corollary 4. If $x$ and $y$ are $\kappa$-adjacent in a $\kappa$-connected digital image $(X, \kappa)$, then $\mathcal{B}_{\kappa}(x, r)$ and $\mathcal{B}_{\kappa}(y, r)$ are $\kappa$-adjacent sets for every nonnegative integer $r$.

3.2. The $\kappa$-neighborhoods as multi-valued functions. Next consider a multivalued function $F: X \multimap Y$ between two digital images $(X, \kappa)$ and $(Y, \lambda)$. That is, $F$ maps each point of $X$ to a subset of $Y$ and for a subset $A$ of $X, F(A)=\cup_{a \in A} F(a)$. The continuity notion for a multi-valued digital map is also defined (see [8-10]) but in this paper we only consider the other two continuity notions given in [27].

Definition 5. [27] Suppose $(X, \kappa)$ and $(Y, \lambda)$ are two digital images and $F: X \multimap$ $Y$ is a multi-valued map.

i): $F$ is said to be weakly continuous, provided whenever $x_{0}$ and $x_{1}$ are $\kappa$ adjacent elements in $X, F\left(x_{0}\right)$ and $F\left(x_{2}\right)$ are $\lambda$-adjacent subsets of $Y$.

ii): $F$ is said to be strongly continuous, provided whenever $x_{0}$ and $x_{1}$ are $\kappa$ adjacent elements in $X$, every point of $F\left(x_{0}\right)$ is $\lambda$-adjacent to some point in $F\left(x_{1}\right)$ and vice versa.

Let $(X, \kappa)$ be a $\kappa$-connected digital image and $r$ be a nonnegative integer. Define the multi-valued function $F_{r}: X \multimap X$ by $F_{r}(x)=\mathcal{B}_{\kappa}(x, r)$ for $x \in X$. Then the following corollary is one of the immediate consequences of Lemma 2.

Theorem 6. The multi-valued function $F_{r}$ is strongly continuous.

Proof. The multi-valued function $F_{0}$ turns into a single-valued function and it satisfies the strong continuity condition immediately. The proof is also trivial when $r=1$ : Let $x_{1}$ and $x_{2}$ be $\kappa$-adjacent in $X$. Since $x_{1} \in \mathcal{B}_{\kappa}\left(x_{2}, 1\right)$, any point of $\mathcal{B}_{\kappa}\left(x_{1}, 1\right)$ is $\kappa$-adjacent to a some point of $\mathcal{B}_{\kappa}\left(x_{2}, 1\right)$ and vice versa. For $r \geq 2$, observe that any element $x \in \mathcal{B}_{\kappa}\left(x_{1}, r\right) \backslash \mathcal{B}_{\kappa}\left(x_{2}, r\right)$ is contained in $\mathcal{B}_{\kappa}\left(x_{2}, r+1\right)$ and this completes the proof.

Corollary 7. $F_{r}$ is weakly continuous.

By Corollary 7, the map $F_{r}$ is also a connectivity preserving function. Note that a multi-valued function $F: X \multimap Y$ is connectivity preserving iff $F$ is weakly continuous and $F(x)$ is a $\lambda$-connected subset of the digital image $(Y, \lambda)$ for every $x \in X[6]$. 
Definition 8. Suppose $(X, \kappa)$ is a $\kappa$-connected digital image and $A$ is a nonempty subset of $X$. For a nonnegative integer $r$, the $r$-thickening of $A, A^{(r, \kappa)}$, is given by

$$
A^{(r, \kappa)}=\cup_{a \in A} \mathcal{B}_{\kappa}(a, r)
$$

Remark 9. $A^{(r, \kappa)}=F_{r}(A)$.

Suppose $\kappa_{1}$ and $\kappa_{2}$ are two adjacency relations on a set $X$. Then we say that $\kappa_{1}$ dominates $\kappa_{2}, \kappa_{1} \geq_{d} \kappa_{2}$, if for $x_{1}, x_{2} \in X$, if $x_{1}$ and $x_{2}$ are $\kappa_{1}$-adjacent then $x_{1}$ and $x_{2}$ are $\kappa_{2}$-adjacent [4]. Further if $X$ is $\kappa_{1}$-(hence $\kappa_{2}$ )-connected and $A$ is a subset of $X$, then $A^{\left(r, \kappa_{1}\right)} \subseteq A^{\left(r, \kappa_{2}\right)}$.

Example 10. Consider the 18-connected digital image $\operatorname{MSS}_{18}=\left\{x_{i}\right\}_{i=0}^{9}$ in $\mathbb{Z}^{3}$ [12] where

$$
\begin{gathered}
x_{0}=(0,0,0), x_{1}=(1,1,0), x_{2}=(0,1,-1), x_{3}=(0,2,-1), x_{4}=(1,2,0), \\
x_{5}=(0,3,0), x_{6}=(-1,2,0), x_{7}=(0,2,1), x_{8}=(0,1,1), x_{9}=(-1,1,0)
\end{gathered}
$$

(see Figure 2). Let $A=\left\{x_{0}, x_{7}\right\}$ and $B=\left\{x_{5}\right\}$. Then $A^{(1,18)}=\mathcal{B}_{18}\left(x_{0}, 1\right) \cup$ $\mathcal{B}_{18}\left(x_{7}, 1\right)=X \backslash\left\{x_{3}\right\}$ and $B^{(2,18)}=X \backslash\left\{x_{0}\right\}$.

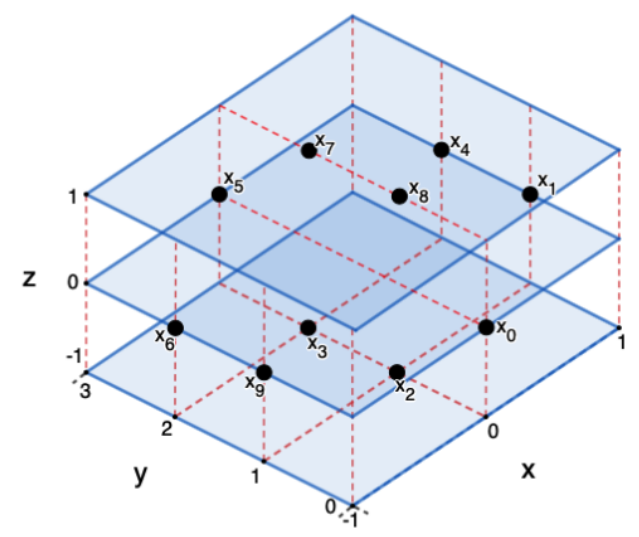

FiguRE 2. 18-connected digital image $\mathrm{MSS}_{18}$ [12].

Proposition 11. Let $(X, \kappa)$ be a $\kappa$-connected digital image and $A$ be a subset of $X$. For two nonnegative integers $r_{1}$ and $r_{2}$, we have $\left(A^{\left(r_{2}, \kappa\right)}\right)^{\left(r_{1}, \kappa\right)}=A^{\left(r_{1}+r_{2}, \kappa\right)}$.

Proof. It is trivial that $A^{\left(r_{1}+r_{2}, \kappa\right)} \subseteq\left(A^{\left(r_{2}, \kappa\right)}\right)^{\left(r_{1}, \kappa\right)}$. Let $a \in\left(A^{\left(r_{2}, \kappa\right)}\right)^{\left(r_{1}, \kappa\right)}$. Then there is $b \in A^{\left(r_{2}, \kappa\right)}$ such that $d^{\kappa}(a, b) \leq r_{1}$ and $c \in A$ such that $d^{\kappa}(b, c) \leq r_{2}$. Therefore

$$
d^{\kappa}(a, c) \leq d^{\kappa}(a, b)+d^{\kappa}(b, c) \leq r_{1}+r_{2}
$$

so $a \in A^{\left(r_{1}+r_{2}, \kappa\right)}$ and this completes the proof. 
The immediate consequence is the following Corollary.

Corollary 12. Suppose $(X, \kappa)$ is a $\kappa$-connected digital image and $A$ and $C$ are $\kappa$ adjacent subsets of $X$. Then there exists $a \in A$ such that $a \in C^{(1, \kappa)}$ or vice-versa.

Proof. The proof follows from Lemma 2

Proposition 13. Suppose $(X, \kappa)$ is a $\kappa$-connected digital image and $A$ is a subset of $X$. Then the following holds:

i): $X \backslash A$ and $A$ are $\kappa$-adjacent,

ii): There exists $x \in X \backslash A$ such that $x \in A^{(1, \kappa)}$.

Proof. : :

i): If $X \backslash A$ and $A$ were not $\kappa$-adjacent sets, this would mean that none of the elements of $X \backslash A$ would be $\kappa$-adjacent to any elements of $A$ so that $X$ would not be $\kappa$-connected.

ii): This follows from $i$ ) and Corollary 12.

Theorem 14. Suppose $(X, \kappa)$ and $(Y, \lambda)$ are digital images and $X$ is $\kappa$-connected. If $A$ is a subset of $X$ and $f: X \rightarrow Y$ is $(\kappa, \lambda)$-continuous, then $f\left(A^{(r, \kappa)}\right) \subseteq f(A)^{(r, \lambda)}$ for every positive integer $r$.

Proof. Let $y \in f\left(A^{(r, \kappa)}\right)$. Then there exists an element $x \in A^{(r, \kappa)}$ such that $f(x)=$ $y$. Let $a$ be a point in $A$ such that $\ell^{\kappa}(x, a) \leq r$; such a point $a$ exists, since $x \in A^{(r, \kappa)}$. That is, the length of the shortest simple $\kappa$-path from $x$ to $a$ in $X$ is less than or equal to $r$. We also have a $\lambda$-path between $y=f(x)$ and $f(a)$ by the continuity of $f$ and the length of this path cannot be greater than $\ell^{\kappa}(x, a)$. If this $\lambda$-path is simple then we have $\ell^{\lambda}(y, f(a)) \leq r$. If it is not simple, we can reduce it to a simple $\lambda$-path from $y$ to $f(a)$ so that the length of the reduced path cannot be greater than $\ell^{\kappa}(x, a)$. Hence $y \in f(A)^{(r, \lambda)}$.

For a digital image $(X, \kappa)$ and its nonempty subset $Y$, we say $Y$ is $\kappa$-dominating in $X$ [7] if for every $x \in X$, there exists $y \in Y$ such that $d^{\kappa}(x, y) \leq 1$. Unlike the definition of $\kappa$-dominating, in the following we consider two subsets of a digital image which need not be contained in one another and we give a notion of $\kappa$ monitoring. For more details on $\kappa$-dominating, see [5].

Definition 15. Let $(X, \kappa)$ be a digital image, $A$ and $B$ be the subsets of $X$. We say that $A \kappa$-monitors $B$ if for any $b \in B$, there exists $a \in A$ such that $d^{\kappa}(a, b) \leq 1$.

Suppose $\kappa_{1}$ and $\kappa_{2}$ are two adjacency relations on a set $X$ such that $\kappa_{2} \geq_{d} \kappa_{1}$ and $A$ and $B$ are subsets of $X$. If $A \kappa_{2}$-monitors $B$, then $A$ also $\kappa_{1}$-monitors $B$.

Remark 16. If $A \kappa$-monitors $B$, then $A$ and $B$ are $\kappa$-adjacent sets. However the converse may not be true. 
Lemma 17. Let $(X, \kappa)$ be a $\kappa$-connected digital image and $A$ and $B$ be the subsets of $X$. Then $A \kappa$-monitors $B$ if and only if $B \subseteq A^{(1, \kappa)}$.

Proof. Let $A \kappa$-monitors $B$ and $b \in B$. Then there is $a \in A$ such that $d^{\kappa}(a, b) \leq 1$ so that $b \in A^{(1, \kappa)}$. This is always true for every element $b$ in $B$, hence $B \subseteq A^{(1, \kappa)}$. On the contrary, let $B \subseteq A^{(1, \kappa)}$. This means that any element $b$ in $B$ is an element in $A^{(1, \kappa)}$ so that there is $a \in A$ such that $d^{\kappa}(a, b) \leq 1$. Therefore $A \kappa$-monitors $B$.

Theorem 18. Let $(X, \kappa)$ and $(Y, \lambda)$ be two digital images, $X$ be $\kappa$-connected, $f$ : $X \rightarrow Y$ be a $(\kappa, \lambda)$-continuous function, and $A$ and $B$ be subsets of $X$. If $A \kappa$ monitors $B$, then $f(A) \lambda$-monitors $f(B)$.

Proof. If $A \kappa$-monitors $B$, then $B \subseteq A^{(\kappa, 1)}$ by Lemma 17. Applying $f$ to that gives $f(B) \subseteq f\left(A^{(\kappa, 1)}\right)$. Since $f\left(\bar{A}^{(\kappa, 1)}\right) \subseteq f(A)^{(1, \lambda)}$ by Theorem 14, we have $f(B) \subseteq f(A)^{(1, \lambda)}$ so that $f(A) \lambda$-monitors $f(B)$.

The following corollary follows from the definition of a strongly continuous multivalued function.

Corollary 19. Let $(X, \kappa)$ and $(Y, \lambda)$ be two digital images, $x_{0}, x_{1} \in X$ and $F: X \multimap$ $Y$ be a strongly continuous multi-valued function. If $x_{0}$ and $x_{1}$ are $\kappa$-adjacent, then $F\left(x_{1}\right) \lambda$-monitors $F\left(x_{0}\right)$ and vice versa.

3.3. Digital Hausdorff Distance. We know that $d^{\kappa}$ is a metric on a $\kappa$-connected digital image $X$. Next, we want to measure the distance between two nonempty subsets $A$ and $B$ of $X$. We call the distance digital Hausdorff distance between them. To do this, we will find the minimum $r$ so that the $r$-thickening of each subset will contain another.

Definition 20. The digital Hausdorff distance between two subsets $A$ and $B$ of a $\kappa$-connected digital image $X$ is

$$
d_{H}^{\kappa}(A, B)=\min \left\{r \geq 0: B \subseteq A^{(r, \kappa)} \text { and } A \subseteq B^{(r, \kappa)}\right\}
$$

Suppose $(X, \kappa)$ is a $\kappa$-connected digital image and $A$ and $B$ are subsets of $X$. If $\kappa_{1}$ is another adjacency relation on $X$ with $\kappa \geq_{d} \kappa_{1}$, then $X$ is also $\kappa_{1}$-connected digital image and $d_{H}^{\kappa}(A, B) \geq d_{H}^{\kappa_{1}}(A, B)$ since the length of the shortest simple $\kappa$-path between any given pair in $X$ might be greater than or equal to the length of the shortest simple $\kappa_{1}$-path between them.

Example 21. Consider the 8-connected digital image $X=\left\{x_{i}\right\}_{i=1}^{6}$ in $\mathbb{Z}^{2}$ where

$$
\left\{x_{1}=(0,0), x_{2}=(0,-1), x_{3}=(1,-1), x_{4}=(1,0), x_{5}=(2,1), x_{6}=(3,1)\right\}
$$


(see Figure 3) and let $A=\left\{x_{1}, x_{2}\right\}$ and $B=\left\{x_{5}, x_{6}\right\}$. Then $d_{H}^{8}(A, B)=3$ since $A \subseteq B^{(2,8)}, B \subseteq A^{(3,8)}$, and $B \nsubseteq A^{(2,8)}$. We also have

$$
d_{H}^{8}\left(F_{1}(A), F_{1}(B)\right)=2
$$

since $F_{1}(A) \subseteq F_{1}(B)^{(1,8)}, F_{1}(B) \subseteq F_{1}(A)^{(2,8)}$, and $F_{1}(B) \nsubseteq F_{1}(A)^{(1,8)}$ where $F_{1}: X \multimap X$ is a multimap defined by $F_{1}(x)=\mathcal{B}_{\kappa}(x, 1)$ for $x \in X$.

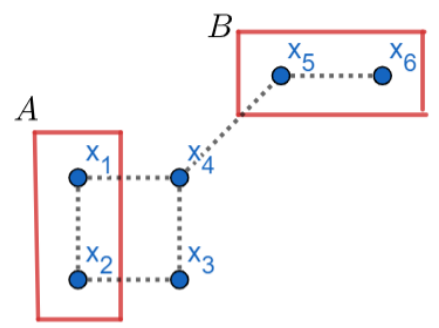

Figure 3. The 8-connected digital image $X$ and the points in red rectanguls from left to right are $A$ and $B$.

Proposition 22. Let $(X, \kappa)$ be a $\kappa$-connected digital image and $A$ be a subset of $X$. Then $d_{H}^{\kappa}(A, A \cup\{x\})=\ell^{\kappa}(x, A)$ for $x \in X$.

Proof. The proof follows from the fact that if $\ell^{\kappa}(x, A)=n$ then $x \in A^{(n, \kappa)}$.

Proposition 23. For a nonnegative integer $r$,

$$
d_{H}^{\kappa}\left(\mathcal{B}_{\kappa}\left(x_{1}, r\right), \mathcal{B}_{\kappa}\left(x_{2}, r\right)\right) \leq 1
$$

whenever $x_{1}$ is $\kappa$-adjacent to $x_{2}$ in a $\kappa$-connected digital image $(X, \kappa)$.

Proof. The proof follows from the fact that $\mathcal{B}_{\kappa}(x, r+1)=\mathcal{B}_{\kappa}(x, r)^{(1, \kappa)}$ for all $x \in X$ and every nonnegative integer $r$.

Now we will prove the stability of the digital Hausdorff distance under a digital continuous map.

Theorem 24. Let $(X, \kappa)$ and $(Y, \lambda)$ be digital images, $X$ connected, $A, B \subseteq X$. If $f: X \rightarrow Y$ is $(\kappa, \lambda)$-continuous, then

$$
d_{H}^{\lambda}(f(A), f(B)) \leq d_{H}^{\kappa}(A, B) .
$$

Proof. Assume that $d_{H}^{\kappa}(A, B)=s$. Then by the definition of the digital Hausdorff distance, $s$ is the minimum number such that $A \subseteq B^{(s, \kappa)}$ and $B \subseteq A^{(s, \kappa)}$. By these inclusions and Theorem 14, we have

$$
\begin{aligned}
f(A) & \subseteq f\left(B^{(s, \kappa)}\right) \subseteq f(B)^{(s, \lambda)} \\
f(B) & \subseteq f\left(A^{(s, \kappa)}\right) \subseteq f(A)^{(s, \lambda)} .
\end{aligned}
$$


so that $f(A)$ and $f(B)$ can be covered by the $s$-thickening of $f(B)$ and $f(A)$ with respect to the adjacency $\lambda$ respectively. Hence $d_{H}^{\lambda}(f(A), f(B)) \leq s$.

Example 25. Consider the following two 4-connected digital images $X=\left\{x_{1}, x_{2}, x_{3}, x_{4}, x_{5}\right\}$ and $Y=\left\{y_{1}, y_{2}, y_{3}\right\}$ in $\mathbb{Z}^{2}$ illustrated in Figure 4. Let $A=\left\{x_{1}, x_{5}\right\}$ and $B=\left\{x_{2}, x_{3}, x_{4}\right\}$ be the two subsets of $X$. If the digital $(4,4)$ continuous map $f: X \rightarrow Y$ is given with

$$
f\left(x_{1}\right)=f\left(x_{4}\right)=y_{2}, f\left(x_{5}\right)=y_{3} \text {, and } f\left(x_{2}\right)=f\left(x_{3}\right)=y_{1},
$$

then $d_{H}^{4}(A, B)=2$ and $d_{H}^{4}(f(A), f(B))=1$.

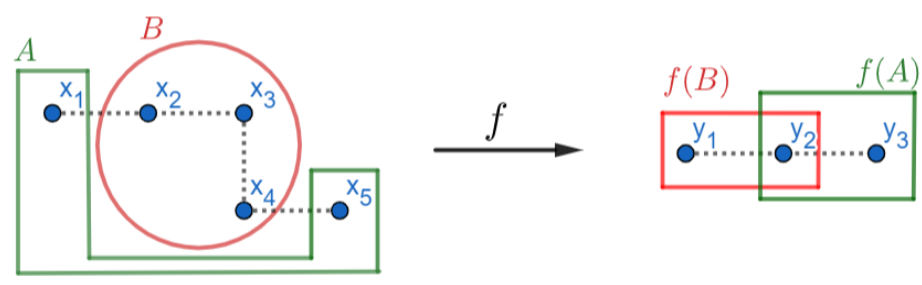

Figure 4. The Hausdorff distance is stable under a digital continuous map between digital images. In this example, we have $d_{H}^{4}(f(A), f(B)) \leq d_{H}^{4}(A, B)$.

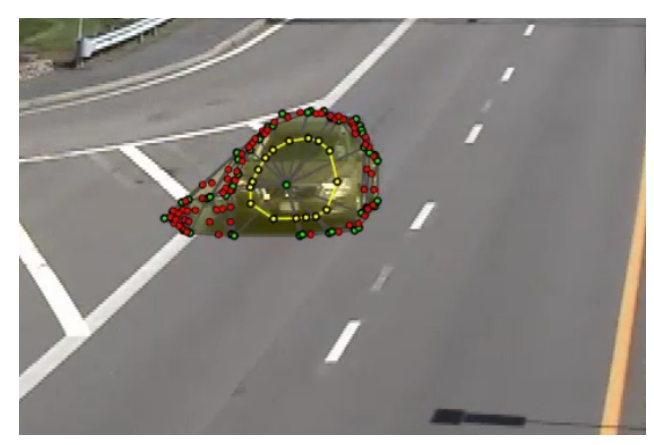

Figure 5. Triangulated traffic video frame shape.

\section{Applichtions}

This section briefly presents applications of Hausdorff Distance. The following two applications were suggested by James F. Peters [21]. 
4.1. Zero-Shot Surface Shape Recognition. This application of the proposed Hausdorff distance between sets focuses on a zero-shot recognition approach in the detection and classification of surface shapes recorded in video frames. Zeroshot classification of images with no training data is highly attractive, since it is less rigid than traditional classification techniques that rely on training data and, hence, build into the learning process unwanted à priori assumptions implicit in the training data. For more about this, see M. Molina and J. Sánchez [19], J. Lu and J. Li and Z. Yan and C. Zhang [18] and J.F. Peters [22].

Let $\operatorname{sh} E, \operatorname{bdy}(\operatorname{sh} E)$ and $\operatorname{int}(\operatorname{sh} E)$ denote a surface shape in a video frame, shape boundary and shape interior, respectively. Also, let $p \in \operatorname{int}(\operatorname{sh} E)$ be the shape centroid and let $t h>0$ be a threshold. For each shape in a video frame, find the Hausdorff distance $D(p, \operatorname{bdy}(\operatorname{sh} E))$ between the centroid of $\operatorname{sh} E$ and $\operatorname{bdy}(\operatorname{sh} E)$ that is less than or equal to a threshold $t h$, defined by

$$
\overbrace{D(p, \operatorname{bdy}(\operatorname{sh} E))=\inf \{\|p-q\|: q \in \operatorname{bdy}(\operatorname{sh} E)\}<t h\}}^{\text {Hausdoff distance criterion }} .
$$

This would be useful in finding video frames that contain shapes that have the required distance property relative to a fixed threshold $t h$.

Example 26. A sample traffic video frame that displays a triangulated vehicle shape shE is shown in Fig. 5. A green vertex $p$ inside the yellow cycle on shE marks the location of the shape centroid. Vertices along the shape boundary are represented by red bullets. The Hausdorff distance $D(p, b d y(s h E))$ would be computed and compared with other vehicle shapes in this video to construct a vehicle shape class.

For a particular shape class, the members of the class satisfy the Hausdorf distance criterion.

4.2. Descriptive Leader Uniform Topology. A clusters form of proximity spacebased uniform topology was introduced by S. Leader [17], elaborated in [24, 25]. This application uses the Hausdorff distance property from Application 4.1 as a feature for a set of video frame shapes equipped with a descriptive proximity mapping $\Phi: \operatorname{sh} E \rightarrow \mathbb{R}$, which provides a basis for the formation of a descriptive Leader uniform class of shapes $\mathfrak{C}_{\Phi}(\operatorname{sh} E)$ for each shape $\operatorname{sh} E$ in the following way.

$$
\begin{aligned}
t h & =\text { selected threshold such that } t h>0 . \\
f r & =\text { video frame. } \\
\operatorname{sh} E \in f r & =\text { video frame shape. } \\
\operatorname{bdy}(\operatorname{sh} E) & =\text { boundary of } \operatorname{sh} E . \\
D(p \in \operatorname{sh} E, \operatorname{bdy}(\operatorname{sh} E)) & =\text { inf }\{\|p-q\|: q \in \operatorname{bdy}(\operatorname{sh} E)\}<t h . \\
\Phi(\operatorname{sh} E) & =D(p \in \operatorname{sh} E, \operatorname{bdy}(\operatorname{sh} E)) . \\
\mathfrak{C}_{\Phi}(\operatorname{sh} E) & =\overbrace{\left\{\operatorname{sh} E^{\prime}:\left\|\Phi(\operatorname{sh} E)-\Phi\left(\operatorname{sh} E^{\prime}\right)\right\|<t h\right\} .}^{\text {Class of video frame shapes }}
\end{aligned}
$$


Then, for each given shape $\operatorname{sh} E$ in a video, a shape $\operatorname{sh} E^{\prime}$ belongs to a class $\mathfrak{C}_{\Phi}(\operatorname{sh} E)$ of shapes relative to $\operatorname{sh} E$, provided $\Phi(\operatorname{sh} E)=\Phi\left(\operatorname{sh} E^{\prime}\right)$ in video frames $f r$ and $f r^{\prime}$ define a descriptive Leader uniform topology, i.e., a collection of shape classes in which nonempty disjoint sets of shapes are descriptively near each other $[23, \S 4.16$, p. 189].

Acknowledgment. This research project has been presented at the 3rd International Conference of Mathematical Sciences (ICMS2019). The author would like to thank James F. Peters for suggesting the applications in Section 4.

\section{REFERENCES}

[1] Boxer, L., Digitally continuous functions, Pattern Recognition Letters, 15 (1994), 833-839.

[2] Boxer, L., A classical construction for the digital fundamental group, Journal of Mathematical Imaging and Vision, 10 (1999), 51-62.

[3] Boxer, L., Multivalued functions in digital topology, Note di Matematica, 37(2) (2017), 61-76.

[4] Boxer, L., Alternate product adjacencies in digital topology, Applied General Topology, 19(1) (2018), 21-53.

[5] Boxer, L., Ege, O., Karaca, I., Lopez, J., Louwsma, J., Digital fixed points, approximate fixed points, and universal functions, Applied General Topology, 17(2) (2016), 159-172.

[6] Boxer, L. Staecker, P. C., Connectivity preserving multivalued functions in digital topology, Journal of Mathematical Imaging and Vision, 55(3) (2016), 370-377.

[7] Chartrand, G., Lesniak, L., Graphs \& digraphs, 2nd ed., Wadsworth, Inc., Belmont, Ca, 1986.

[8] Escribano, C., Giraldo, A., Sastre, M., Digitally continuous multivalued functions, in Discrete Geometry for Computer Imagery, Lecture Notes in Computer Science, v. 4992, 81-92, Springer, 2008.

[9] Escribano, C., Giraldo, A., Sastre, M., Digitally continuous multi-valued functions, morphological thinning algorithms, Journal of Mathematical Imaging and Vision, 42(1) (2012), 76-91.

[10] Giraldo, A., Sastre, M., On the composition of digitally continuous multivalued functions, Journal of Mathematical Imaging and Vision, 53 (2) (2015), 196-209.

[11] Han, S.-E., Non-product property of the digital fundamental group, Information Sciences, 171(1) (2005), 73-91.

[12] Han, S.-E., Digital fundamental group and Euler characteristic of a connected sum of digital closed surfaces, Information Sciences, 177 (2007), 3314-3326.

[13] Han, S.-E., Estimation of the complexity of a digital image from the viewpoint of fixed point theory, Applied Mathematics and Computation, 347 (2019), 236-248.

[14] Han, S.-E., Digital k-Contractibility of an n-Times Iterated Connected Sum of Simple Closed k-Surfaces and Almost Fixed Point Property, Mathematics, 8(345) (2020), 1-24, doi:10.3390/math8030345

[15] Herman, G.T., Oriented surfaces in digital spaces, CVGIP: Graphical Models and Image Processing, 55 (1993), 381-396.

[16] Khalimsky, E., Motion, deformation and homotopy in finite spaces, In Proceedings IEEE International Conference on Systems, Man, and Cybernetics, (1987), 227-234.

[17] Leader, S., On clusters in proximity spaces, Fund. Math., 47 (1959), 205-213.

[18] Lu, J., Li, J., Yan, Z., Zhang, C., Zero-Shot Learning by Generating Pseudo Feature Representations, arXiv1703.06389v1, 2017, 1-9. 
[19] Molina, M., Sánchez, J., Zero-Shot Learning with Partial Attributes, Intelligent Computing Systems. ISICS 2018. Communications in Computer and Information Science, vol 820, Springer Nature, Switzerland AG, (2018), 147-158, https://doiorg.uml.idm.oclc.org/10.1007/978-3-319-76261-6\_12.

[20] Munkres, J., Topology (2nd ed.), pp. 280-281, Prentice Hall, 1999.

[21] Peters, J.F., personal communication.

[22] Peters, J.F., Computational geometry, topology and physics of digital images with applications. Shape complexes, optical vortex nerves and proximities, Springer Nature, Cham, Switzerland, 2020, xxv+440 pp., https://doi-org.uml.idm.oclc.org/10.1007/978-3-030-221928, Zbl07098311.

[23] Peters, J.F., Topology of Digital Images. Visual Pattern Discovery in Proximity Spaces, Intelligent Systems Reference Library 134 (2014), Springer Heidelberg, xxvii+ 433pp, https://doiorg.uml.idm.oclc.org/10.1007/978-3-642-53845-2, Zentralblatt MATH Zbl 129568010.

[24] Peters, J.F., Computational proximity. Excursions in the topology of digital images, Intelligent Systems Reference Library 102 (2016), Cham: Springer, xv + 411pp, https://doiorg.uml.idm.oclc.org/10.1007/978-3-319-30362-4, Zentralblatt MATH Zbl 1382.68008.

[25] Peters, J.F., Proximal Voronoï regions, convex polygons, \& Leader uniform topology, Adv. Math.: S.J., 4 (1) (2015), 1-5, Zbl 1335.65032.

[26] Rosenfeld, A., Continuous functions on digital images, Pattern Recognition Letters, 4 (1987), $177-184$.

[27] Tsaur, R. and Smyth, M. B., "Continuous" multifunctions in discrete spaces with applications to fixed point theory, In: Bertrand G., Imiya A., Klette R. (eds) Digital and Image Geometry, Lecture Notes in Computer Science, vol 2243 Springer, Berlin, Heidelberg, 2001. 